\title{
The Evaluation of basic and neurohormonal parameters in hot or cold temperament person proposed in Iranian Traditional Medicine: an observational study
}

\author{
Gholamreza Mohammadi Farsani, ${ }^{a}$ Mohsen Naseri, Saeed Hosseini, ${ }^{b}$ Ali Akbar Saboor-Yaraghi, ${ }^{c}$ Mohammad \\ Kamalinejad, ${ }^{\mathrm{d}}$ Taiebeh Mohammadi Farsani, ${ }^{\mathrm{e}}$ Ahmadreza Dorosti Motlagh, ${ }^{\mathrm{f}}$ Mina Movahhed ${ }^{9}$
}

\begin{abstract}
'Department of Clinical Nutrition, School of Nutritional Sciences and Dietetics, Tehran University of Medical Sciences, Tehran, Iran. Minimally Invasive Surgery Research Center, Iran University of Medical Sciences, Tehran, IR Iran. Mohammadigh53@gmail.com

2Iranian Traditional Medicine Clinical trial Research Center, Shahed University, Tehran, Iran. naserishahed@yahoo.com

${ }^{3}$ Department of Clinical Nutrition, School of Nutritional Sciences and Dietetics, Tehran University of Medical Sciences, Tehran, Iran. saeedhmdphd@hotmail.com

${ }^{4}$ Department of Immunology, School of Public Health, Tehran University of Medical Sciences, Tehran, Iran. asaboor@tums.ac.ir

${ }^{5}$ Department of Pharmacognosy, School of Pharmacy, Shahid Beheshti University of Medical Sciences, Tehran, Iran. mkamalinejad@yahoo.com

${ }^{6}$ Department of Medical Biotechnology, Isfahan (Khorasgan) Branch Islamic Azad University, Isfahan, Iran, Minimally Invasive Surgery Research Center, Iran University of Medical Sciences, Tehran, IR Iran. tmfarsani@gmail.com

`Department of Clinical Nutrition, School of Nutritional Sciences and Dietetics, Tehran University of Medical Sciences, Tehran, Iran. A_Dorosty@yahoo.com

${ }^{8}$ Traditional Medicine and Materia Medica Center, Shahid Beheshti University of Medical Sciences, Tehran, Iran. Movehhedm54@gmail.com

*Corresponding authors Mina Movahhed: Traditional Medicine and Materia Medica Center, Shahid Beheshti University of Medical Sciences, No.8 Shams Alley, Vali-e-Asr Street, Tehran, Iran, P.O.Box 1516745811, Tel/Fax: 98-21-8877 6027, Mobile: +98-9120617507 Email: movahhedm54@gmail.com

Ahmadreza Dorosti Motlagh: Address: Department of Clinical Nutrition, School of Nutritional Sciences and Dietetics, Tehran University of Medical Sciences, Tehran, Iran. P.O Box 6446 Tehran 14155, I.R. Iran, Tel: +98 (21) 88992710, Cell: +98 9129727934 Email: A_Dorosty@yahoo.com

Author contributions: Gholamreza Mohammadi Farsani (GMF): Study design, statistical analysis, drafted the manuscript. Mohsen Naseri (MN): Study design Saeed Hosseini (SH): Study design Masoud Yunesian (MY): Study design Ali Akbar Saboor-Yaraghi (AAS): Study design Mohammad Kamalinejad (MKN): Study design, statistical analysis. Taiebeh Mohammadi Farsani (TMF): assisted to draft the manuscript. Mina Movahhed (MM): Study design, assisted to draft the manuscript Ahmadreza Dorosti Motlagh (ADM): conceived the study, Study design, statistical analysis.
\end{abstract}

\begin{abstract}
Objective: Much of energy for life sustainment is consumed as the basal metabolic rate (BMR). Temperament is one of the core concepts in Iranian Traditional Medicine. The aim of this study is to assess the BMR and activity of the sympathetic-parasympathetic nervous system and thyroid function in hot and cold temperament people. Our study is a repeated cross-sectional study which was implemented in 2 stages on 45 healthy volunteers.

Methods: BMR was evaluated by indirect calorimetry. Thyroid function, sympathetic-parasympathetic nervous system activity, body composition, and nutrition status were also evaluated. We used independent $t$-test for data analysis by SPSS ver. 16.

Results: Overall, 45 patients aged 18-40 participated in this study. Our results showed that the mean of BMRs were respectively 1664.09 and $1909.48 \mathrm{kcal}$ in cold and hot temperament individuals $(P<0.3)$. Systolic and diastolic blood pressure, and heart rate peripheral temperature of individuals with hot temperament were significantly higher $(P<0.05)$, While no statistically significant difference was seen in norepinephrine to epinephrine ratio, norepinephrine to cortisol ratio and core temperature. T3 and TSH levels were respectively, 1.99 and 1.26 in cold temperament individuals and 1.38 and 1.40 in hot temperament individuals $(P<0.05)$.

Conclusion: It shows signs that there is a relationship between the BMR and neurohormonal system and body temperament meaning cold temperament people have lower BMR, sympathetic nervous system activity and thyroid function in comparison to others. Body health is negatively affected by BMR fluctuations. With regarding to this correspondence, the arrangement of a pattern for better BMR regulation is crucial.

Keywords: Temperament; Iranian Traditional Medicine; Basal Metabolic Rate; Sympathetic - Parasympathetic nervous System; Thyroid function

Abbreviations: Basal Metabolic Rate (BMR), Iranian Traditional Medicine (ITM), Thermic Effect of Food (TEF), Activity Thermogenesis (AT), Bioelectrical Impedance Analysis (BIA), Enzyme-Linked Immunosorbent Assay (ELISA).
\end{abstract}

\section{Introduction}

The total energy consumed by human body consists of basal or resting metabolic rate (BMR), thermic effect of food (TEF), and activity thermogenesis (AT). BMR is the minimum amount of expended energy that is compatible with life and it is needed to sustain the metabolic activities of cells and tissues as well as to maintain the processes of circulatory, respiratory, gastrointestinal, and renal systems. ${ }^{1,2}$ BMR comprises approximately $60-70 \%$ of the total energy expenditure which is affected by several factors such as age, sex, body composition, size, heredity and molecular genetics, hormones, and environmental conditions. ${ }^{3-6}$
Some hormonal factors including the autonomous nervous system and thyroid hormones affect BMR. As one of the most important factors affecting BMR, sympathetic-parasympathetic nervous system is influenced by various factors such as age, sex, stress, psychological, and emotional status, etc. Moreover, the thyroid function modulates the BMR and following conditions such as cold and heat tolerance, fatigue, euphoria, heart rate, movement speed, etc. ${ }^{7}$ According to the preceding issues, differences in BMR can be a result of diversities of factors affecting the 
sympathetic-parasympathetic nervous system and thyroid function. ${ }^{8-17}$

Iranian Traditional Medicine (ITM, Persian medicine) is one of the oldest schools of traditional medicine. ${ }^{18-22}$ From ITM point of view, temperament is one of the basic concepts of health and disease. Temperament is variable among individuals and is affected by several factors such as age, sex, environment, and body composition..$^{23-26}$

Recent investigations have shown that hot (warm) and cold temperaments can be associated with BMR and similarly with sympathetic-parasympathetic nervous system activity. ${ }^{27}$. ${ }^{28}$ Some of these relationships are shown in Table 1.

According to the foregoing discussion, it seems that hot temperament people have a higher BMR in comparison to cold temperament people. A new window is hoped to be created in nutrition science and public health by development of BMR concept in traditional medicine viewpoint. In order to investigate this relationship, comparison between the BMR of both cold and hot temperament individuals was carried out.

\section{Materials and Methods}

This is a descriptive-analytic study. Since the under investigation variables can be affected by different factors, all the variables were investigated in 2 stages with a 2-4 weeks interval.

In order to select individuals undergoing the study, a notification was published and distributed among the students residing in Tehran University of Medical Sciences dormitories.

\section{Inclusion Criteria}

Age from 18 to 40 years, healthy individuals (according to history and physical examination), normal body mass index, informed consent.

\section{Exclusion Criteria}

Use of any specific dietary pattern such as vegetarianism, smoking, alcohol imbibing, or regular consumption of coffee, Taking any medications or supplements in the past month, abnormal biochemical lab test after evaluation, not having tendency to participate in the study.

In this study, demographic characteristics such as height, weight, vital signs, blood group, RH, BMR, body composition, and biochemical assays (measurement of serum levels of adrenaline, noradrenalin, cortisol, T3, T4, and TSH) were evaluated. Nutrient intake was assessed using a food frequency questionnaire and three 24 -h dietary recalls.

Temperaments of participants were determined by a validated questionnaire ${ }^{29}$ and the expert diagnosis (traditional medicine specialist). Based on the foresaid temperament determining questionnaire, if the total score of the individual was 19 or greater, he was hot temperament; and if it was 14 or less, the temperament was cold. If the score's range was from 15 to 18 , the individual's temperament was considered mild that was excluded from the study. The number of gender-divided hot and cold temperament participants was based on required sample size.

BMR was measured by the use of indirect calorimetry (METALYZER 1B). In order to control the factors affecting metabolic rate, avoidance of any food especially coffee and cigarette 12 hours before the test was recommended to the participants. They should be also inhibited from moderate and severe aerobic or anaerobic activity 2 and 14 hours before the test, respectively. Before performing indirect calorimetry, the participants should rest for 10-20 min. During the test, the room temperature was maintained $20-25^{\circ} \mathrm{C} .{ }^{30}$ Participants' body composition, including weight, fat, water and muscle percentage, and fat-free mass were measured by Bioelectrical Impedance Analysis (BIA) (Company INBODY Model 770).

Serum thyroid function tests were measured using Enzyme-Linked Immunosorbent Assay (ELISA) and "PishtazTebcompany kits", epinephrine and norepinephrine using 2-CAT ELISA kit manufactured by Immunobiological Laboratories company, cortisol by DCMO23-4 kit by DiaMetra company, and blood group and RH by Cinnaclone II kit.

Calculations were performed using $80 \%$ power, a $5 \%$ significance level, and a $25 \%$ dropout rate. The required sample size was approximately 40 participants for each group allowing a $25 \%$ withdrawal rate.

\begin{tabular}{|c|c|c|}
\hline Variable & Basal metabolic rate & Temperament \\
\hline Age & $\begin{array}{l}\text { *High in the first and second year of life } \\
{ }^{*} \text { Reduces } 2-3 \% \text { in each decade of life-1,2 }\end{array}$ & $\begin{array}{l}\text { *Hot temperament is more common in children and the } \\
\text { young } \\
{ }^{*} \text { Temperament heat starts to decrease after the age of } 35-40^{5}\end{array}$ \\
\hline sex & $\begin{array}{l}\text { In the same weight and height, BMR in } \\
\text { women, is } 5-10 \% \text { less than men }{ }^{1,2}\end{array}$ & Female temperament is colder than the male $\mathrm{e}^{5}$ \\
\hline Body composition & $\begin{array}{l}\text { *Muscle mass is the most significant } \\
\text { determinant of BMR. } \\
\text { *It increases BMR } R^{1,2}\end{array}$ & $\begin{array}{l}\text { *High muscle mass indicate shot temperament } \\
\text { *High fat mass indicates cold temperament }{ }^{4}\end{array}$ \\
\hline Body surface & $\begin{array}{l}\text { Those who have more body surface, have } \\
\text { higher BMR' }\end{array}$ & $\begin{array}{l}\text { *High muscle mass indicate shot temperament } \\
\text { *High fat mass indicates cold temperament }{ }^{4}\end{array}$ \\
\hline $\begin{array}{l}\text { The sympathetic- } \\
\text { parasympathetic nervous system }\end{array}$ & $\begin{array}{l}\text { Stimulating the sympathetic nervous } \\
\text { system (e.g. stress) raises the BMR' }\end{array}$ & $\begin{array}{l}\text { Some emotional conditions can increase body's hot } \\
\text { temperament }{ }^{6}\end{array}$ \\
\hline Thyroid function & $\begin{array}{l}\text { *Hypothyroidism reduces the BMR } \\
\text { *Hyperthyroidism increases the BMR, }\end{array}$ & $\begin{array}{l}\text { *Individuals with cold temperament feel cold more than } \\
\text { others \& tolerate heat better than the cold in normal } \\
\text { conditions. } \\
{ }^{*} \text { In hot temperament individuals, it is reversed }\end{array}$ \\
\hline
\end{tabular}


We used SPSS (Statistical Package for Social Sciences, ver 16) program for Statistical analysis. Mean and standard deviation were calculated for quantitative variables and frequency (percent) describes qualitative variables. Independent $t$-test was used to compare BMR, thyroid function and body composition, nutrient intake, clinical characteristics, and biochemical tests between two groups. Less than $P<0.05$ was assumed as significance.

This study was approved by Tehran University of Medical Sciences ethics committee bearing code number 90-04-2715366-55307. All subjects were informed through a written consent form.

\section{Results}

Totally, 45 patients aged $18-40$ years participated in this study. Two people were excluded because of not being able to complete the study. Finally, 43 patients terminated the study, 20 of whom were male (46.5\%) and 23 were females (53.5\%). Ten men had hot temperament and 10 had cold temperament. Among women, 11 had hot temperament and 12 had cold temperament.

The average age of the participants was $27.47 \pm 4.65$ years that this average was more in cold temperament people, but no statistically significant difference existed between them $(P$ value $=0.152)$. Hematocrit and hemoglobin levels were in the normal range in all participating subjects and no statistically significant differences existed among the two hot and cold temperament people (hot and cold groups) ( $P$ value $>0.05)$, But the amounts of hemoglobin and hematocrit were significantly higher in men $(P$ value $<0.05)$.

The results of the anthropometric indices showed that weight, height, and body mass index had no significant diversity among the two groups ( $P$ value $>0.05)$.

As shown in Table 2, body fat mass and percentage were significantly greater in the cold groups $(P$ value $<0.05)$ and the percentage of fat free mass and skeletal muscle mass was significantly higher in the hot group $(P$ value $<0.05)$. Although the amount and percentage of body water was more in cold group, this difference was not statistically significant $(P$ value $>0.05)$

\section{Nutrient Intake Results}

Based on FFQ, intake of energy (2572.2 vs $1900.61 \mathrm{kcal}$ ), carbohydrate ( 361.40 vs 253.24 ), fat ( 89.61 vs 70.08 ) and protein (94.33 vs $76.24 \mathrm{~g}$ ) was significantly higher in the hot group ( $P$ value $<0.05)$.
Based on the 24-h recall questionnaire, the individuals' food intake temperament was assessed relatively due to the expert idea in both days before intervention. Based on the results, no significant relationship existed among the two groups in any days before intervention ( $P$ value $>0.05)$.

\section{Biochemical and Metabolic Findings}

As shown in Table 3, the hot group has a significantly higher BMR $(P$ value $=0.03)$ even after adjusting BMR for weight $(P$ value $=.006)$. T3 amounts were significantly higher in hot group and TSH was higher in cold group $(P$ value $<0.05)$. Although T4 rate was higher in hot group, it can't be considered meaningful $(P$ value $=0.457)$.

As shown in Fig 1, heart rate, systolic and diastolic blood pressure, and peripheral temperature of the hot group was significantly higher $(P$ value $<0.05)$. But core temperature, the norepinephrine to epinephrine and norepinephrine to cortisol ratio were not significantly diverse among them $(P$ value $>0.05$ )

\section{Discussion}

The results indicate that body composition of people with hot temperament significantly differs from people with cold temperament; So that fat tissue is more in cold group and the percentage of fat-free mass and skeletal muscle mass is more in hot group. These findings confirm the traditional medicine viewpoint about the temperament. According to this view, the temperament of fat tissue is cold and muscle tissue is hot. Accordingly, the amount of fat is higher in the body of cold group, while the amount of muscle is higher in the body of hot group. In conclusion, body composition can be used as a marker to identify individuals' temperaments.

Based on the findings of this study, no significant relationship was seen between the blood group or Rh and temperament which may be due to small sample size. For exact assessment of this relation and further studies, greater sample sizes are needed in this regard. Our results indicate that BMR varies in hot and cold group. Indeed, the mean BMR is higher in hot group. Even after weight adjusting (the metabolic rate per kilogram of body weight), the similar results were seen. Basal metabolism maintenance and preventing it from slowing down or rising up would affect human health. Although traditional concept of temperament is not adverted in modern medicine, investigation of factors influencing temperament indicates that it's possible 


\begin{tabular}{|c|c|c|c|c|c|}
\hline \multirow[t]{3}{*}{ Variable } & \multicolumn{4}{|c|}{ Temperament of individuals } & \multirow{3}{*}{$\begin{array}{c}\text { Significance statistical } \\
\text { test*P value }\end{array}$} \\
\hline & \multicolumn{2}{|c|}{ Cold temperament $\mathrm{n}=22$} & \multicolumn{2}{|c|}{ Hot temperament $\mathbf{n}=21$} & \\
\hline & Mean & Standard deviation & Mean & Standard deviation & \\
\hline Body fat mass(kg) & 13.41 & 2.15 & 18.35 & 4.15 & $.001>$ \\
\hline Body fat percentage & 21.73 & 5.47 & 27.98 & 7.37 & .003 \\
\hline Skeletal muscle mass (kg) & 30.99 & 7.15 & 27.03 & 5.48 & .047 \\
\hline Fate free mass percentage & 78.49 & 5.72 & 72.02 & 7.37 & .003 \\
\hline Body water (Lt) & 36.14 & 8.34 & 37.41 & 6.47 & .579 \\
\hline Body water percentage & 55.70 & 6.23 & 56.11 & 462 & .807 \\
\hline
\end{tabular}

Independent $t$-test*

\begin{tabular}{|c|c|c|c|c|c|}
\hline \multirow[t]{3}{*}{ Variable } & \multicolumn{4}{|c|}{ Temperament of individuals } & \multirow{3}{*}{$\begin{array}{l}\text { Significance statistical } \\
\text { test*P value }\end{array}$} \\
\hline & \multicolumn{2}{|c|}{ Cold temperament $\mathrm{n}=22$} & \multicolumn{2}{|c|}{ Hot temperament $n=21$} & \\
\hline & Mean & SD & Mean & SD & \\
\hline Basal metabolic rate/Kgbw(kcal) & 25.74 & 2.96 & 28.76 & 3.88 & .006 \\
\hline Basal metabolic rate(kcal) & 1664.09 & 252.04 & 1909.48 & 442.40 & .030 \\
\hline T3 & 1.26 & .13 & 1.40 & .18 & .008 \\
\hline T4 & 7.19 & .48 & 7.29 & .34 & .457 \\
\hline TSH & 1.99 & 1.24 & 1.38 & .63 & .049 \\
\hline
\end{tabular}

to consider these effective factors for BMR regulation. In ITM, many health protecting orders and diagnostic and therapeutic procedures are determined based on the temperament of individuals. Temperament of each individual is based on several physical and psychological characteristics which are completely introduced in ITM sources Temperament detecting templates have diagnostic roles in determining the temperament. Based on ITM view, different reactions to pathogenic conditions can be explained by temperament consideration so new abilities may be built to forecast diseases related to temperament susceptibility and to improve treatments on the basis of food and drug temperament. Regarding the differences between nutrient intake of hot and cold group, according to the results of our study, it seems that hot group by virtue of having a higher BMR and more muscle tissue, has a greater need for energy and as a result, total calorie and following different macronutrients intake is higher in them. In order to prove the relation between the sympatheticparasympathetic nervous system and temperament, the clinical indices and vital signs were also measured in addition to plasma epinephrine, norepinephrine, and cortisol level which were assessed in Shahabi et al. study. Epinephrine and norepinephrine are indices for assessing the balance between environmental sympathetic nervous system and adrenal sympathetic while norepinephrine to cortisol ratio is an index of balance between environmental sympathetic and parasympathetic activity.
Clinical indices include heart rate, systolic blood pressure, diastolic blood pressure, core temperature, and the ambient temperature. Based on the results of this study, the norepinephrine to epinephrine ratio and norepinephrine to cortisol ratio is not significantly different between hot and cold groups.

The differences between results of this study and the study of Shahabi et al may be due to the difference in the time of measurement. Our sampling was carried out early in the morning and in the fasting state while in the study of Shahabi et al, the sampling was implemented at 3-5 PM. As so many factors are effective in the balance of the sympathetic-parasympathetic system, more appropriate indicators may be needed to measure this amount.

The clinical parameters and vital signs of the individuals were meaningfully different in two groups. The results of this study showed that heart rate, systolic blood pressure, diastolic blood pressure, and ambient temperature were significantly higher in hot group verifying more sympathetic activity in the hot temperament and more parasympathetic activity in cold temperament. Similarly symptoms of hypothyroidism in cold temperament and hyperthyroidism signs in hot temperament suggest their relationship with each other in the mind. The results of our study confirm this hypothesis and show that there is a direct correlation between temperament and the level of thyroid function. 


\section{Conclusion}

It seems that there is a relationship between the BMR and neurohormonal system and body temperament meaning hot temperament people have higher BMR, sympathetic nervous system activity, and thyroid function in comparison to others. Human health is negatively affected by BMR fluctuations. Regarding this correspondence, arrangement of a pattern for better BMR regulation is crucial.

Obviously, more studies are needed in order to make the two schools of medicine closer to each other in their path stepping forward health maintenance and disease control.

Furthermore, coldness of temperament may cause fat accumulation in body. This may probably imply that body fat mass is decreased by temperament heating.

\section{Acknowledgments}

This study was supported by the School of Nutritional Sciences and Dietetics, Tehran University of Medical Sciences. The school of Pharmacy, Shahid Beheshti University of Medical Sciences, the School of Traditional Medicine, Shahed University of Medical Sciences and Noorafshar Rehabilitation and Sports Medicine Hospital provided this study with cooperation. The authors reported no conflicts of interest.

\section{Conflict of interest disclosure:}

The authors reported no conflicts of interest.

\section{Funding:}

This study was funded by Tehran University of Medical Sciences, Tehran, Iran.

\section{References:}

1. McMurray RG, Soares J, Caspersen CJ, McCurdy T. Examining variations of resting metabolic rate of adults: a public health perspective. Med Sci Sports Exercise. 2014;46(7):1352.

2. Manini TM. Energy expenditure and aging. Ageing research reviews. 2010;9(1):1-11

3. Carol D, Rachel K, Mahan L. Escott Stump S. Krause's food \& nutrition therapy 12e. Canada: Saunders El-Sevier; 2008

4. White CR, Kearney MR. Determinants of inter-specific variation in basal metabolic rate. J Comp Physiol B. 2013;183(1):1-26.

5. Swanson DL, McKechnie AE, Vézina F. How low can you go? An adaptive energetic framework for interpreting basal metabolic rate variation in endotherms. J Comp Physiol B. 2017:1-18

6. Feng $P$, Zhao H, Lu X. Evolution of mitochondrial DNA and its relation to basal metabolic rate. Mitochond DNA. 2015;26(4):566-71.

7. McCrory P, Strauss B, Wahlqvist ML. Energy balance, food intake and obesity. Exercise and obesity London: Smith Gordon. 1994:115-30.

8. SL. J. Alaghraz Altebieh and Mabahes Allaeieh. Tehran University of Medical Sciences, Iran2005.

9. Khorasani SA. Kholasatol Hekmah. Medicine 1st vol Qom: Ismaielian. 2006:52-146.
10. Sina Al. Al-Qanun fi al-tibb2005

11. Zhou X, Mao D, Luo J, Wu J, Zhuo Q, LiY. Study of Basal metabolic rate of 8 young adults aged 20-29 years old in Changsha. Zhonghua yu fang yi xue za zhi [Chin J Prev Med]. 2017:51(7):642-6.

12. Ali N, Mahmood S, Manirujjaman M, Perveen R, Nahid A, Ahmed S, et al. Hypertension prevalence and influence of basal metabolic rate on blood pressure among adult students in Bangladesh. BMC Public Health. 2018;18(1):58.

13. Boratyński JS, Jefimow M, Wojciechowski MS. Individual differences in the phenotypic flexibility of basal metabolic rate in Siberian hamsters are consistent on short-and long-term timescales. Physiol Biochem Zool. 2017;90(2):139-52.

14. Lee HJ, Yang SJ. Aging-related correlation between serum sirtuin 1 activities and basal metabolic rate in women, but not in men. Clin Nutr Res. 2017;6(1):18-26.

15. Anthanont $P$, Jensen MD. Does basal metabolic rate predict weight gain? Am J Clin Nutr. 2016;104(4):959-63.

16. Maximov AL, Belkin VS, Kalichman L, Kobyliansky ED. Adaptive changes in basal metabolic rate in humans in different eco-geographical areas. Colleg Antropol. 2015;39(4):887-92.

17. Nilsson JF, Nilsson JÅ. Fluctuating selection on basal metabolic rate. Ecol Evol. 2016:6(4):1197-202

18. Rezadoost H, Karimi M, Jafari M. Proteomics of hot-wet and cold-dry temperaments proposed in Iranian traditional medicine: A Network-based Study. Scientific reports. 2016;6:30133

19. Parvinroo S, Kamalinejad M, Sabetkasaei M. Pharmacological concepts of temperament in Iranian Traditional Medicine. Iran J Public Health. 2014;43(10):1463-5

20. Emami M, Nazarinia MA, Rezaeizadeh H, Zarshenas MM. Standpoints of traditional Persian physicians on geriatric nutrition. J Evid-based Complem Altern Med. 2014:19(4):287-91.

21. Parvinroo S, Naghibi F, Zahediasl S, Kamalinejad M, Sabetkasaei M. The effects of seeds with hot and cold temperaments on serum thyroid hormones, corticosterone and urine vanillylmandelic acid concentrations of healthy rats. J Ethnopharmacol. 2014;156:216-21.

22. Rezaeizadeh H, Alizadeh M, Naseri M, Ardakani MS. The traditional Iranian medicine point of view on health. Iran J Publ Health. 2009;38(1):169-72.

23. Alizadeh $M$, Khadem E, Aliasl J. Diagnosis protocol of stomach distemperament for clinical practice in iranian traditional medicine: A narrative review. Iran J Public Health. 2017;46(7):877.

24. Zeinalian M, Eshaghi M, Hadian M, Naji H, Marandi SMM, Asgary S. Eight essential foods in Iranian traditional medicine and their role in health promotion and well-being. Int J Prev Med. 2017;8.

25. Miraj S, Alesaeidi S, Kiani S. A systematic review of the relationship between dystemprament (sue Mizaj) and treatments and management of diseases (Ilaj and Eslah-e-Mizaj). Electr Phys. 2016;8(12):3378.

26. Kopaei R, Khajegir A, Kiani S. The association between dystemperament and prevention of diseases: A systematic review. J Clin Diag Res: JCDR. 2016:10(9):YE01.

27. Farsani GM, Movahhed M, Motlagh AD, Hosseini S, Yunesian M, Farsani TM, et al. Is the Iranian Traditional Medicine warm and cold temperament related to Basal Metabolic Rate and activity of the sympatheticparasympathetic system? Study protocol. J Diab Metab Disord. 2014;13(1):74.

28. Shahabi S, Hassan ZM, Mahdavi M, Dezfouli M, Rahvar MT, Naseri M, et al. Hot and cold natures and some parameters of neuroendocrine and immune systems in traditional Iranian medicine: A preliminary study. J Altern Complement Med. 2008:14(2):147-56.

29. Mojahedi M, Naseri M, Majdzadeh R, Keshavarz M, Ebadini M, Nazem E, et al. Reliability and validity assessment of Mizaj questionnaire: a novel self-report scale in Iranian traditional medicine. Iranian Red Crescent Med J. 2014:16(3).

30. Compher C, Frankenfield D, Keim N, Roth-Yousey L, Group EAW. Best practice methods to apply to measurement of resting metabolic rate in adults: A systematic review. J Am Diet Assoc. 2006;106(6):881-903.

This work is licensed under a Creative Commons Attribution-NonCommercial 3.0 Unported License which allows users to read, copy, distribute and make derivative works for non-commercial purposes from the material, as long as the author of the original work is cited properly. 